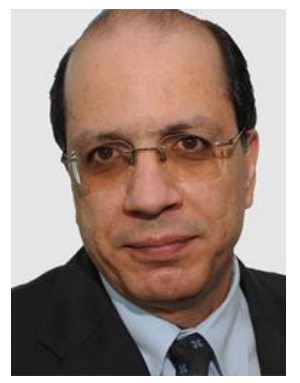

\title{
Substantial Research Secures the Blue Future for our Blue Plant
}

\author{
Prof. Moustafa Abdel-Maksoud \\ Director, Institute for Fluid Dynamics and Ship Theory \\ Hamburg University of Technology (TUHH), Germany
}

Earth, the blue planet, is our home, and seas and oceans cover more than $70 \%$ of its surface. As the earth's population rapidly increases and available resources decrease, seas and oceans can play a key role in assuring the long-term survival of humankind. Renewable maritime energy has huge potential to provide a considerable part of the earth's population with decarbonised electricity generation systems. Renewable maritime energy is very flexible and can be harvested above the water's free surface by using offshore wind turbines, on the water's surface by using wave energy converters or below the water's surface by using current or tidal turbines. The supposed conflict between environmental protection measures and economic interests is neither viable nor reasonable. Renewable maritime energy can be the motor for considerable substantial economic growth for many maritime regions and therefore for society at large.

The fastest growing sector of renewable maritime energy is offshore wind. The annual report of the European Wind Energy Association from the year 2015 confirms the growing relevance of the offshore wind industry. In 2015, the total installed and grid-connected capacity of wind power was $12,800 \mathrm{MW}$ in the EU and 6,013.4 MW in Germany. 38\% of the 2015 annual installation in Germany was offshore, accounting for a capacity of 2,282.4 MW. However, there are a limited number of available installation sites in shallow water, meaning that there is an urgent need to develop new offshore structures for water depths greater than $50 \mathrm{~m}$. The persistent trend towards deeper waters has encouraged the offshore wind industry to look for floating wind turbine structures and larger turbines.

Floating wind turbine technologies are at an early stage of development and many technical and economic challenges will still need to be faced. Nonetheless, intensive research activities and the employment of advanced technologies are the key factors in accelerating the design process and ensuring the safe operation of floating wind turbines. In order to optimise the overall system, interdisciplinary numerical simulation methods are essential for evaluating the dynamic structure's behaviour. Due to the motion of the floating structure, the wind turbine design and the power generation system must be optimised to withstand high accelerations. Advanced control systems are crucial for maintaining a safe operation of the system.

Reducing costs is a main challenge for the offshore wind industry. One strategy in this effort is to assemble floating structure and the wind turbine onshore and then towing them out to sea. This procedure eliminates the need to use installation jack-up vessels with high crane capacity and therefore reduces overall installation time and costs. Floating structures are designed to accommodate large turbines; therefore, it should be expected that the final cost per MWh will be lower.

Another important resource of renewable maritime energy is wave energy. It has a considerable extracting potential and is available nearly up to 90 per cent of the time, which is not the case for wind and solar energy. However, there is no converged solution for the best method of extracting wave energy. Research in this area is still in a very early stage as compared to wind or solar energy technologies.

The main challenge in designing wave energy converters (WEC) is the strong fluctuation of the available 
wave power levels, which can vary by a factor of 100 . This means a WEC has to work efficiently at a low energy level and withstand extreme wave conditions that can occur once every few years. That is not only a structural problem but it is also a design problem with many challenges with respect to optimising the hydrodynamic behaviour of the system and the dynamic characteristics of the power take-off systems. The efficiency of a WEC can be significantly increased by using an adaptive active control of the power take-off. Current research focuses on the performance of the overall system, which includes developing new concepts for the WEC as well as the individual subcomponents of the system. This research is currently ongoing for developing advanced control approaches for efficiently extracting energy, and for developing concepts to minimise energy losses via the transmission of the slow reciprocating motion of the floating bodies into the rotary motion of the power generator and power transmission system.

Another attractive renewable maritime energy source is the tidal stream, the intensity of which has the benefit of being accurately predicted far in advance. Tidal energy is the oldest renewable maritime energy, with tide mills being used by the eighth century C.E. in many countries along the European west coast. Tides are very long waves, which take place twice a day and induce a movement of a huge amount of water. High current velocities can occur when the water enters certain confined areas such as bays or between two neighbouring islands. The tidal energy can be harnessed in such coastal areas using tidal turbine devices which are designed to extract kinetic energy from the tidal stream. The tidal turbine designs are very similar to wind turbines except that the rotor is driven by the tidal current. The rotor can be mounted on a founded or floating structure. As the density of water is more than 800 times higher than air, the diameter required of a turbine rotor to achieve a certain power is much smaller than a wind turbine rotor. Similar to a WEC, there is no converged solution for the best method of extracting wave energy but in the last years a number of promising concepts have been presented that can be further developed. While implementing reliable tidal turbine designs with low installation and operation costs still requires more time and research, tidal turbines are an attractive solution for some coastal locations far from the public electricity net, such as small islands or holiday regions.

In some coastal regions, the geographical conditions allow for building a near shore tidal dam to accumulate tidal water. The water enters the dam at high tide and leaves it at low tide. The tidal energy can be harnessed by using conventional water turbines which convert the hydraulic power into electric power. The turbines in this case are well developed and therefore only some minor improvement are necessary. The cost of constructing the tidal dam is high; however, the operation costs are relatively low. A tidal dam can have a strong Influence on aquatic life and therefore the environmental impacts of such a project must be investigated very accurately.It is therefore essential to intensify the research and development activities on renewable maritime energy technology to make the vision of a blue future for our blue planet to become reality.

\section{About Prof. Moustafa Abdel-Maksoud}

Professor Moustafa Abdel-Maksoud is the head of the Institute for Fluid Dynamics and Ship Theory at Hamburg University of Technology in Germany. He is well-known for his contributions to a wide range of hydrodynamic analysis and experiments in the area of naval architecture and ocean engineering. His recent research interests include ship hydrodynamics and dynamics; design and optimization of ship propulsion systems; Fluid-Structure Interaction and Renewable Maritime Energy. Prof. Maksoud is the leader developing panMARE in-house panel code with wide industry applications. He has leaded and coordinated various national and EU projects. The most relevant project in the renewable energy area is Maritime Safety Aspects Regarding Installation and Maintenance of Offshore Wind Turbines supported by Research and Science Foundation Hamburg.

Prof. Moustafa Abdel-Maksoud obtained a B.Sc. in Marine Engineering and Naval Architecture at Alexandria University in 1982. He took his M.Sc. at the same, and then studied for his doctorate which he obtained in 1992 at the TU Berlin. Following research positions and headship at Potsdam Model Basin, he became Professor at the Institute of Ship Technology and Transport systems, Duisburg-Essen University in 2003, and subsequently became Head of Institute of Fluid dynamics and Ship Theory, TUHH in 2007. Prof. Abdel-Maksoud is a member of numerous professional associations. 